\title{
The Extended Sech Function Method and The Cole- Hopf Transformation for Solving the Nonlinear Korteweg-de Vries Equation
}

\author{
M. M. El-Horbaty ${ }^{1}$, F. M. Ahmed ${ }^{2}$ \\ ${ }^{1}$ Mathematics Department, Faculty of Science, Zagazig University, Egypt \\ ${ }^{1,2}$ Mathematics Department, Faculty of Science, Alejelat, Zawia University, Libya
}

\begin{abstract}
In this paper, we are using the extended sech function method along with a type of cole-Hopf transformation to obtain the solutions for the nonlinear Korteweg -de Vries (KdV) equation. These types of solutions are represented as the hyperbolic function solutions including the solitary wave solution, shock wave solution and trigonometric function solution when the modulus $m$ approaches to 1 and 0 . Mathematica software is used in calculation and graphics.
\end{abstract}

Keywords: Cole-Hopf transformation, Extended Sech function, Korteweg -de Vries (KdV) equation, Solitary travelling wave solutions

\section{Introduction}

It is well known that the Korteweg - de Vries equation is the generic outcome of a weakly nonlinear long-wave asymptotic analysis of many physical systems. It is categorized by its family of solitary wave solutions, with the familiar " $\mathrm{Sech}^{2}$ " profile. However, in those circumstances when they are two wave modes with nearly coincident linear long wave speeds. Nonlinear Partial differential equations (PDE's) have a crucial role in various Scientific fields. The nonlinear wave phenomena such as dispersion, dissipation, diffusion... etc, are essential in nonlinear wave equations. Those waves are normally kink shaped with tanh-solutions and bell shaped with the sech-solutions. Last decades, a variety of powerful methods are proposed and established for obtaining an explicit solitary traveling wave solution of the nonlinear PDE's [4], [9], [23]-[25], [29] who are interested in nonlinear physical phenomena. Tanh-sech methods[1], [6], [7]. extended tanh method [11]. For integrable nonlinear differential equations, the inverse scattering transformation method [3], the Hirota method [9], the truncated painlevé expansion method [23], the Backlund transformation method [15], the exp-Function method and the Sech method [2]-[7], [10], [17], are used in looking for exact solution. There are many different methods to look for the exact solutions of those equations.

In the presented work, we implemented the Extended sech function method and the Cole-Hopf transformation to obtain the solitary travelling wave solutions of the nonlinear Korteweg -de Vries equation $(\mathrm{KdV})$ of the form:

$$
u_{t}+\alpha u u_{x}+\mu u_{x x x}=0
$$

Where $\alpha$ and $\mu$ are real constants and to be determined later.

\section{Description of the used Method}

2.1 The Methodology of The Extended Sech function method

The extended sech function method proposes that a given nonlinear Partial differential equation (PDE) in one dimension of the form:

$$
\boldsymbol{\Phi}\left(u, u_{t}, u_{x}, u_{x x}, \ldots\right)=0
$$

is transformed into a nonlinear ordinary differential equation (ODE) (3), using the wave variable $\xi=k x-\omega t$ so that $u(x, t)=U(\xi)$. Therefore:

$$
\boldsymbol{\Psi}\left(U, U^{\prime}, U^{\prime \prime}, U^{\prime \prime \prime}, \ldots\right)=0
$$

A new independent variable is introduced of the form :

$$
Y=\operatorname{sech}(\xi)
$$

The first and all higher derivatives is derived similarly to be:

$$
\begin{aligned}
\frac{d \bullet}{d \xi} & =Y \sqrt{1-Y^{2}} \frac{d \bullet}{d Y} \\
\frac{d^{2} \bullet}{d \xi^{2}} & =Y\left[\left(1-2 Y^{2}\right) \frac{d \bullet}{d Y}+Y\left(1-Y^{2}\right) \frac{d^{2} \bullet}{d Y^{2}}\right] \\
\frac{d^{3} \bullet}{d \xi^{3}} & =Y \sqrt{1-Y^{2}}\left[\left(1-6 Y^{2}\right) \frac{d \bullet}{d Y}+\right. \\
& \left.+3 Y\left(1-2 Y^{2}\right) \frac{d^{2} \bullet}{d Y^{2}}+Y^{2}\left(1-Y^{2}\right) \frac{d^{3} \bullet}{d Y^{3}}\right]
\end{aligned}
$$

The extended sech function method admits the solution of (3) takes the form:

$$
u(x, t)=U(\xi)=\sum_{i=0}^{n} a_{i} Y^{i}(\xi)+\sum_{i=1}^{n} b_{i} Y^{-i}(\xi)
$$

Then we define the degree of $U(\xi)$ as $D[U(\xi)]=n$, which gives rise to the degree of other expression as 


\section{International Journal of Science and Research (IJSR) \\ ISSN (Online): 2319-7064}

Index Copernicus Value (2013): 6.14 | Impact Factor (2015): 6.391

$D\left\lfloor\frac{d^{\prime} U}{d \xi^{\prime}}\right\rfloor=n+r, D\left\lfloor\left(\frac{d^{\prime} U}{d \xi^{\prime}}\right)^{4}\right\rfloor=q(n+r)$ and

$D\left\lfloor U^{\prime}\left(\frac{d^{\prime} U}{d \xi^{\prime}}\right)^{\prime}\right\rfloor=n p+q(n+r)$. Thus we can obtain the value of

$n$ in (6) by balancing the derivative term of the highest order with the nonlinear term. Substituting (6) into (3) and setting all coefficients of $Y^{i}, Y^{-i}$ to zero, an algebraic system for the unknown coefficients $a_{i}$ and $b_{i}$ is generated and to be solved. Consider, the $\mathrm{KdV}$ equation (1) and using $\xi=k x-\omega t$, $u(x, t)=U(\xi)$ with the change of derivatives (5). The obtained ODE takes the form:

$$
-\omega U^{\prime}+\alpha k U^{2} U^{\prime}+\mu k^{3} U^{\prime \prime \prime}=0
$$

All terms in the ODE (7) contain derivatives, for simplicity, it is integrated so that the constant of integration is set to be zero, gives:

$$
-\omega U+\frac{\alpha k}{3} U^{3}+\mu k^{3} U^{\prime \prime}=0
$$

Balancing the highest order derivative $\left(U^{\prime \prime}\right)$ with nonlinear term ( $\left.U^{3}\right)$ in (8) gives $n=2$. Thus, (6) admits the expansion:

$$
U(\xi)=a_{0}+a_{1} Y(\xi)+a_{2} Y^{2}(\xi)+b_{1} Y^{-1}(\xi)+b_{2} Y^{-2}(\xi)
$$

Substituting (9) into (8) the following algebraic system is obtained for $Y^{-i}(\xi), \quad-4 \leq i \leq 4$ :

$$
\begin{aligned}
\frac{1}{2} k \alpha b_{2}^{2} & =0, \\
k \alpha b_{1} b_{2} & =0, \\
\frac{1}{2} k \alpha b_{1}^{2}+4 k^{3} \mu b_{2}-\omega b_{2}+k \alpha a_{0} b_{2} & =0, \\
k^{3} \mu b_{1}-\omega b_{1}+k \alpha a_{0} b_{1}+k \alpha a_{1} b_{2} & =0, \\
-\omega a_{0}+\frac{1}{2} k \alpha a_{0}^{2}+k \alpha a_{1} b_{1}-2 k^{3} \mu b_{2}+k \alpha a_{2} b_{2} & =0, \\
k^{3} \mu a_{1}-\omega a_{1}+k \alpha a_{0} a_{1}+k \alpha a_{2} b_{1} & =0, \\
\frac{1}{2} k \alpha a_{1}^{2}+4 k^{3} \mu a_{2}-\omega a_{2}+k \alpha a_{0} a_{2} & =0, \\
-2 k^{3} \mu a_{1}+k \alpha a_{1} a_{2} & =0, \\
-6 k^{3} \mu a_{2}+\frac{1}{2} k \alpha a_{2}^{2} & =0
\end{aligned}
$$

The parameters $k, \omega, \alpha$ and $\mu$ are assumed to be nonzero, solitary wave solutions are built with the solution of (10), by aid of Mathematica software:

$$
\begin{aligned}
& \omega=-4 k^{3} \mu, a_{2}=\frac{12 k^{2} \mu}{\alpha}, a_{0}=-\frac{8 k^{2} \mu}{\alpha}, \\
& b_{2}=a_{1}=b_{1}=0
\end{aligned}
$$

$$
\omega=4 k^{3} \mu, a_{2}=\frac{12 k^{2} \mu}{\alpha}, b_{2}=a_{0}=a_{1}=b_{1}=0
$$

Returning the values of $\omega, a_{0}, a_{1}, a_{2}, b_{1}, b_{2}$ from (11) and (12) into (9), the solitary wave solutions are built in the following forms:

$$
\begin{aligned}
& U(\xi)=-\frac{8 k^{2} \mu}{\alpha}+\frac{12 k^{2} \mu}{\alpha} Y(\xi)^{2}, \quad \xi=k x+4 \mu k^{3} t \\
& U(\xi)=\frac{12 k^{2} \mu}{\alpha} Y(\xi)^{2}, \quad \xi=k x-4 \mu k^{3} t
\end{aligned}
$$

Taking in consideration (4) The graphics of the obtained surfaces in (13) and (14) are presented in figure.2 and figure. 3 respectively, for given values.

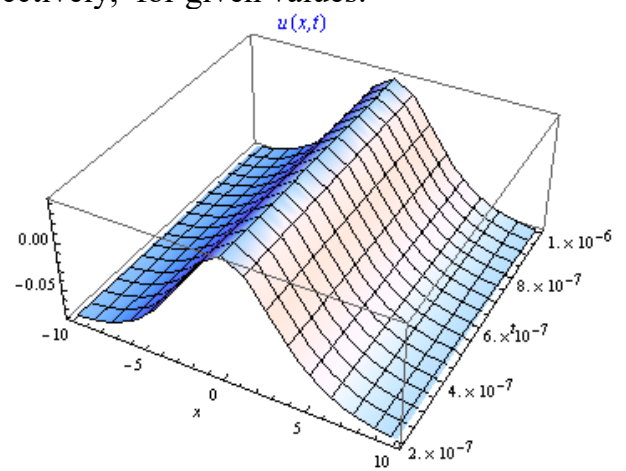

Figure 1 The Extended Sech Function of (13) with $\alpha=6, k=0.25, \mu=1,-10 \leq x \leq 10$ and $2 \times 10^{-7} \leq t \leq 10 \times 10^{-7}$.

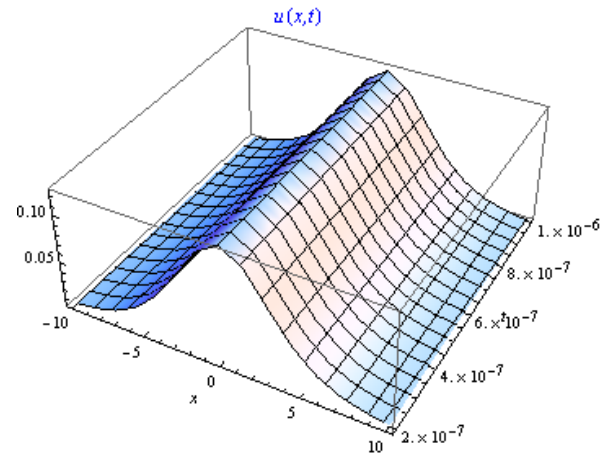

Figure 2 The Extended Sech Function of (14) with $\alpha=6, k=0.25, \mu=1,-10 \leq x \leq 10$ and $2 \times 10^{-7} \leq t \leq 10 \times 10^{-7}$.

\subsection{The Cole Hopf Transformation and the analytical solution of the $\mathrm{KdV}$ equation}

In this section, we discuss the Korteweg - de Vries (KdV) equation (1) which is transformed into the nonlinear ordinary differential equation (ODE) after integrating with zero constant of integration, to become:

$$
-\omega U^{\prime}+\alpha k U U^{\prime}+\mu k^{3} U^{\prime \prime \prime}=0
$$

Integrating with respect to $\xi$ and taking into account that the integration constant to be zero, the ordinary differential equation (15) takes the following form;

$$
p U-q U^{2}-r U^{\prime \prime}=0
$$

Where : 


\section{International Journal of Science and Research (IJSR) \\ ISSN (Online): 2319-7064}

Index Copernicus Value (2013): 6.14 | Impact Factor (2015): 6.391

$$
p=\omega, \quad q=\frac{\alpha k}{2}, \quad r=\mu k^{3}
$$

Consider the Cole-Hopf transformation :

$$
U(\xi)=\frac{\partial^{2}}{\partial \xi^{2}} \operatorname{Ln} G=G^{-1} G_{\xi \xi}-G^{-2} G_{\xi}^{2}
$$

The first and the second derivatives would have the form:

$$
\begin{aligned}
U^{\prime}= & G^{-1} G_{3 \xi}-3 G^{-2} G_{\xi} G_{\xi \xi}+2 G^{-3} G_{\xi}^{3} \\
U^{\prime \prime}= & G^{-1} G_{4 \xi}-4 G^{-2} G_{\xi} G_{3 \xi}-3 G^{-2} G_{\xi \xi}^{2} \\
& +12 G^{-3} G_{\xi}^{2} G_{\xi \xi}-6 G^{-4} G_{\xi}^{4}
\end{aligned}
$$

Substituting by the transformation $U(\xi)$, (18), and its derivatives (19) and (20). The Equation in (16) is reduced to the following:

$$
\begin{aligned}
& p\left(G^{-1} G_{\xi \xi}-G^{-2} G_{\xi}^{2}\right)+q\left(G^{-1} G_{\xi \xi}-G^{-2} G_{\xi}^{2}\right)^{2} \\
& +r\left(G^{-1} G_{4 \xi}-4 G^{-2} G_{\xi} G_{3 \xi}-3 G^{-2} G_{\xi \xi}^{2}\right. \\
& \left.+12 G^{-3} G_{\xi}^{2} G_{\xi \xi}-6 G^{-4} G_{\xi}^{4}\right)=0
\end{aligned}
$$

The power classification for $G^{i}, \quad-1 \leq i \leq-4$ is to be:

$$
\begin{aligned}
G^{-1}: & p G_{\xi \xi}-r G_{4 \xi}=0 \\
G^{-2}: & -p G_{\xi}^{2}+(-q+3 r) G_{\xi \xi}^{2}-4 r G_{\xi} G_{\xi \xi \xi}=0 \\
G^{-3}: & 2(-q+6 r) G_{\xi}^{2} G_{\xi \xi}=0 \\
G^{-4}: & (q-6 r) G_{\xi}^{4}=0
\end{aligned}
$$

From (24) and (25) we obtain the condition $q=6 r$, which leads to the undetermined nonlinear coefficient:

$$
\alpha=12 \mu k^{2}
$$

From (22) by integrating twice with considering the integrating constants equal to zero, to take the form:

$$
G_{\xi \xi}-\lambda^{2} G=0, \quad \lambda^{2}=\frac{p}{r}=\frac{\omega}{\mu k^{3}}
$$

Its solution is:

$$
G=c_{1} e^{\lambda \xi}+c_{2} e^{-\lambda \xi}, \quad \lambda=\sqrt{\frac{\omega}{\mu k^{3}}}
$$

Thus,

$$
U(\xi)=\frac{G G_{\xi \xi}-G_{\xi}^{2}}{G^{2}}=\frac{4 c_{1} c_{2} \lambda^{2}}{\left(c_{1} e^{\lambda \xi}+c_{2} e^{-\lambda \xi}\right)^{2}}
$$

Let $\frac{c_{2}}{c_{1}}=m^{2}, \quad 0<m^{2} \leq 1$, then (29) becomes, the JacobiGlaisher functions for elliptic function which can be found [5], [16] :

$$
\begin{aligned}
U(\xi) & =\frac{4 \lambda^{2} m^{2}}{\left(e^{\lambda \xi}+m^{2} e^{-\lambda \xi}\right)^{2}}, \\
& m=\sqrt{\frac{c_{2}}{c_{1}}}, \quad \lambda=\sqrt{\frac{\omega}{\mu k^{3}}}, \quad \alpha=12 \mu k^{2}
\end{aligned}
$$

Noting that as a modulus $m \rightarrow 1$, the solution of the Korteweg-de Vries equation tends to the hyperbolic function $\operatorname{sech}^{2}(\lambda \xi)$.

A graphical representation of the solution (30) is shown in figure .3 , figre. 4 and figure. 5 for some values of the modulus $m$

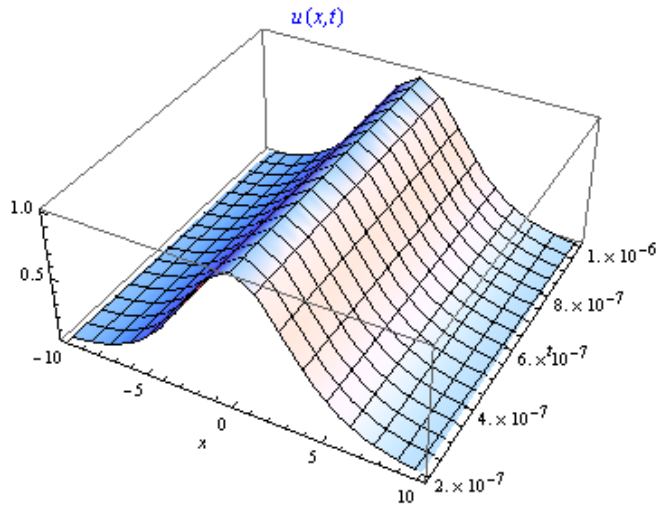

Figure 3: The Cole- hopf surface (30) with $m=1, k=0.25, \mu=1, \alpha=0.75, \omega=0.015625-10 \leq x \leq 10$ and $2 \times 10^{-7} \leq t \leq 10 \times 10^{-7}$.

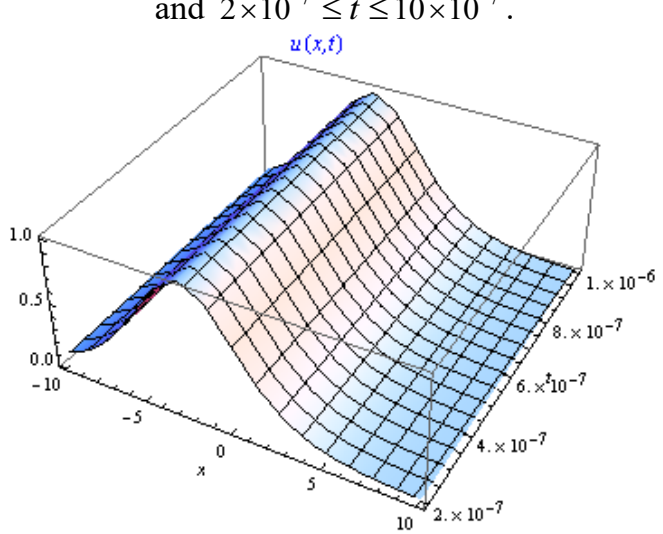

Figure 4 The Cole- hopf surface (30) with $m=0.5, k=0.25, \mu=1, \alpha=0.75, \omega=0.015625$

$-10 \leq x \leq 10$ and $2 \times 10^{-7} \leq t \leq 10 \times 10^{-7}$.

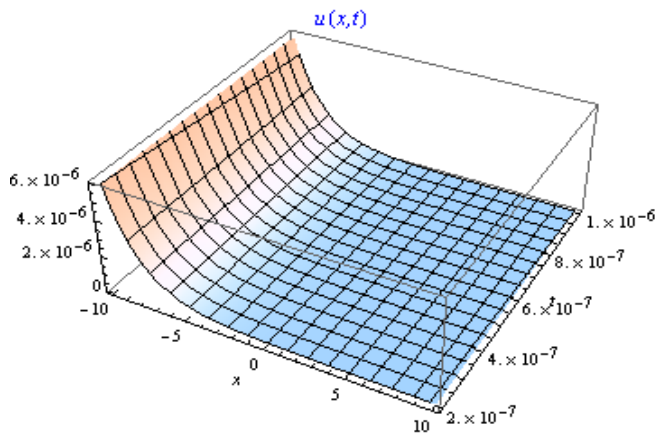

Figure 5 The Cole- hopf surface (30) with $m=0.0001, k=0.25, \mu=1, \alpha=0.75, \omega=0.015625$ $-10 \leq x \leq 10$ and $2 \times 10^{-7} \leq t \leq 10 \times 10^{-7}$. 


\section{International Journal of Science and Research (IJSR) \\ ISSN (Online): 2319-7064}

Index Copernicus Value (2013): 6.14 | Impact Factor (2015): 6.391

\section{Conclusion}

In this study, we employed the extended sech function method and the Cole-Hopf transformation for finding the solitary travelling wave solutions of the Korteweg -de Vries equation (KdV). The methods have been shown to computationally efficient in solving the equation of our interest. The last observation is that, with an appropriate value of the modulus $m$ in (30) the solution tends to the hyperbolic function sech. By means of Mathematica all Graphics and computations are achieved .

\section{A Appendix A : Properties of the Jacobi elliptic functions}

I. They satisfy The identities: $\quad \operatorname{cn}^{2} \xi+\operatorname{sn}^{2} \xi=1$,

$$
\mathrm{dn}^{2} \xi+m^{2} \operatorname{sn}^{2} \xi=1
$$

II. Derivatives of the Jacobi elliptic functions

$$
\operatorname{sn}^{\prime} \xi=\operatorname{cn} \xi \operatorname{dn} \xi, \mathrm{cn}^{\prime} \xi=-\operatorname{sn} \xi \operatorname{dn} \xi,
$$$$
\mathrm{dn}^{\prime} \xi=-m^{2} \operatorname{sn} \xi \mathrm{cn} \xi \text {. }
$$

Where $m$ is a modulus. The Jacobi-Glaisher functions for elliptic function can be found in [5], [16].

III. A modulus $m$ tends to one gives, $\lim _{m \rightarrow 1} \operatorname{sn} \xi=\tanh \xi$,

$$
\begin{aligned}
& \lim _{m \rightarrow 1} \operatorname{cn} \xi=\operatorname{sech} \xi, \lim _{m \rightarrow 1} \operatorname{dn} \xi=\operatorname{sech} \xi, \text { and for } m \\
& \text { tends to zero we get } \lim _{m \rightarrow 0} \operatorname{sn} \xi=\sin \xi, \\
& \lim _{m \rightarrow 0} \operatorname{cn} \xi=\cos \xi, \quad \lim _{m \rightarrow 0} \operatorname{dn} \xi=1
\end{aligned}
$$

\section{References}

[1] Abdu M. A. , The extended tanh-method and its applications for solving nonlinear physical models. Appl. Math. Comput. 2007;(190) 988-96.

[2] Abdu M. A. , The extended F-expansion method and its applications for a class of nonlinear evaluation equation chaos solitons. Fractals 2007; (31) 95-104

[3] Ablowitz M J and Clarkson, Nonlinear Evolution Equations and Inverse scattering transform, P A 1991 Solitons, Cambridge : Cambridge university press.

[4] Bekir A. and Boz. A., Exact solutions for nonlinear evaluation equations using exp-function method, Phys. Lett. A. 2008; (372) 1619-25.

[5] Chandrasekharan K. Elliptic functions. Berlin: Springer; 1978 .

[6] El-Wakil SA, Abdou MA, New exact travelling wave solutions using modified extended tanh-function method. Chaos Solitons Fractals2007; 31(4); 840- 52.

[7] Fan. E. G., Extended tanh-function method and its applications to nonlinear equations, Phy. Lett. A. 2000;(277) 212-18.

[8] He J. H. and Wu X. H, Exp-function method for nonlinear wave equations, Chaos Solitons Fractals 2006; (30) 700-8.

[9] Hirota R., Exact solution of the KdV equation for multiple collisions of solitons, Phys. Rev. Lett. $1971 ;(27)$ 1192-4.
[10] Inc M, Ergut M, Periodic wave solutions for the generalized shallow water wave equation by the improved Jacobi elliptic function method. Appl Math ENotes 2005; 5; 89-96.

[11] Khater AH, Malfliet W, Callebaut DK and Kamel ES, The tanh method, a simple transformation and exact analytical solutions for nonlinear reaction diffusion equations. Chaos Solitons Fractals 2002; 14(3); 513-22.

[12] Kudryashov N. A., On types of nonlinear nonintegrable equations with exact solutions, Phys. Lett. A. 1991; (155) 269-75.

[13] Malfliet W, Solitary wave solutions of nonlinear wave equations. Am JPhys 1992; 60(7); 650- 4.

[14] Miura M. R., Backlund Transformation 1978 (Berlin: Springer).

[15] Monro S, Parkes EJ, Stability of solitary-wave solutions to a modified Zakharov-Kuznetsov equation.J Plasma Phys 2000; 64(3), 411- 426.

[16]Patrick Dv. Elliptic Function and elliptic curves. Cambridge: University press; 1973.

[17] Sheng Zhang, The periodic wave solutions for the $(2+$ 1)-dimensional Konopelchenko Dubrovsky equations. Chaos Solitons Fractals 2006; 30; 1213- 20.

[18] Wang M. and Li X., Extended F-expansion and periodic wave solutions for the generalized Zakharov equations, Phys. Lett. A 2005, (343) 48-54.

[19] Wazwaz AM, Two reliable methods for solving variants of the KdV equation with compact and non compact structures. Chaos Solitons Fractals2006; 28(2); 454- 62.

[20] Wazwaz AM, The tanh-function method: Solitons and periodic solution for the Dodd-Bullough-Mikhailov and the Tzitzeica-Dodd-Bullough equations. Chaos Solitons and Fractals 2005; 25(1); $55-63$.

[21] Wazwaz AM, The sine-cosine method for obtaining solutions with compact and non- compact structures. Appl Math Comput 2004; 159(2); 559-76.

[22] Wazwaz AM, A sine-cosine method for handling nonlinear wave equations. Math Comput Modelling 2004; 40(5-6); $499-508$.

[23] Weiss J, Tabor $M$ and Garnevalle G, The painlevé property for partil differential equations. J. Math. Phys. 24 942-7

[24]Xia TC, Li B and Zhang HQ, New explicit and exact solutions for the Nizhnik- Novikov-Vesselov equation. Appl Math E-Notes 1 2001; 139-42.

[25] Yusufoglu E. and Bekir, Exact solution of coupled nonlinear evaluation equations, Chaos Solitons Fractals A 2008; (37) 842-8.

[26] Yusufoglu E, Bekir A, Solitons and periodic solutions of coupled nonlinear evolution equations by using SineCosine method. Internat J Comput Math 2006; 83(12); 915-24.

[27]Yusufoglu E, BekirA, On the extended tanh method applications of nonlinear equations. International Journal of Nonlinear Science 2007; $1 ; 10-16$.

[28]Zayed EME, Zedan H. A. and Gepreel K. A. ,Group analysis and modified tanh-function to find the Invariant solutions and soliton solutions for nonlinear Euler Equations. International Journal of nonlinear Sciences and Numerical Simulation. 2011; 5 (3) 221-234.

[29] Zayed EME, Abourabia AM, Gepreel K. A. and ElHorbaty M. M., Travelling solitary wave solution for the 


\section{International Journal of Science and Research (IJSR) \\ ISSN (Online): 2319-7064}

Index Copernicus Value (2013): 6.14 | Impact Factor (2015): 6.391

nonlinear coupled Korteweg -de Vries system, Chaos Solitons and Fractals 2007; (34) 296-06

[30]Zhang SL, ,WUB and Lousy, The painlevé analysis and special solutions of generalized Broer-Kaup equations. Phys. Lett, 2002; A 300 40-8 